\title{
Micelle Density Regulated by a Reversible Switch of Protein Secondary Structure
}

Rory E. Sallach, ${ }^{1}$ Min Wei, ${ }^{2}$ Nilanjana Biswas, ${ }^{3}$ Vincent P. Conticello, ${ }^{4}$ Sébastien Lecommandoux, ${ }^{5}$ Richard A. Dluhy, ${ }^{3}$ and Elliot L. Chaikof ${ }^{2,6^{*}}$

${ }^{1}$ Department of Biomedical Engineering, Georgia Institute of Technology, Atlanta GA 30332, ${ }^{2}$ Departments of Surgery and Biomedical Engineering, Emory University School of Medicine, ${ }^{3}$ Department of Chemistry, University of Georgia, Athens GA, ${ }^{4}$ Department of Chemistry, Emory University, Atlanta, GA 30322, ${ }^{5}$ CNRS Laboratory of Organic Polymer Chemistry, University Bordeaux 1, Talence Cedex, France. and ${ }^{6}$ School of Chemical and Biomolecular Engineering, Georgia Institute of Technology, Atlanta, GA 30332

Submitted as a SUPPORTING INFORMATION for a Research Article to the

Journal of the American Chemical Society

\author{
$\dagger$ Address correspondence to: \\ Elliot L. Chaikof, M.D., Ph.D. \\ E-mail: echaiko@emory.edu \\ Emory University \\ 101 Woodruff Circle, Rm 5105 \\ Atlanta, GA 30322 \\ Tel: (404) 727-8413 \\ Fax: (404)-727-3660
}




\section{CONTENTS}

Experimental methods and general procedures

S3-S8

Dynamic light scattering measurements

S9-S11 


\section{EXPERIMENTAL SECTION}

Synthesis of B9. Synthetic methods used to produce the DNA inserts that encode the various elastin block copolymers have been described previously. ${ }^{26,27}$ Oligonucleotide cassettes encoding the elastic $(\mathbf{A})$ and plastic $(\mathbf{B})$ repeat units (Table I) were independently synthesized and inserted into the BamH I/HinD III sites within the polylinkers of pZErO-1 and pZErO-2, respectively (Scheme 1). Recombinant clones were isolated after propagation in E. coli strain Top10F', double-stranded DNA sequence analysis verified the identity of the DNA inserts A and B. These clones were propagated in E. coli strain SCS110 in order to isolate preparative amounts of plasmid DNA. DNA monomers A and $\mathbf{B}$ were liberated from the respective plasmids via restriction digestion with $B s p$ M I and SexA I, respectively. Selfligation of each DNA cassette afforded a population of concatemers encoding repeats of the plastic and elastic sequences, respectively.

Concatemers derived from DNA monomer $\mathbf{B}$ were inserted into the BspM I site of the modified polylinker $\mathbf{C}$ in plasmid pET-24a. A concatemer encoding sixteen repeats of the plastic sequence was isolated and identified via restriction cleavage with Kpn I and Pst I. Double-stranded DNA sequence analysis confirmed the integrity of the concatamer within the recombinant plasmid, which was labeled $\mathrm{pPC}$. The concatemer was liberated from $\mathrm{pPC}$ via restriction cleavage with Kpn I and Pst I and purified via preparative agarose gel electrophoresis. Enzymatic ligation was used to join the concatemer cassette to the Kpn I/Pst I sites within the modified polylinker D in plasmid pET-24a. Double stranded DNA sequence analysis confirmed the integrity of the concatemer within the recombinant plasmid, which was labeled pPN. The pair of recombinant plasmids pPN and pPC encoded the $N$-terminal and $C$ terminal domains, respectively. Restriction cleavage of each plasmid with SexA I and Xma I afforded two fragments, which were separated via preparative agarose gel electrophoresis. 
Enzymatic ligation of the large fragment of $\mathrm{pPN}$ and the small fragment of $\mathrm{pPC}$ afforded the recombinant plasmid $\mathrm{pP} 2$ as a single contiguous reading frame within plasmid pET-24a.

Plasmid pP2 was propagated in E. coli strain SCS110 and cleaved with restriction endonuclease SexA I. Concatemers encoding the elastin sequence A were inserted into the compatible SexA I site of $\mathrm{pP} 2$. The recombinant clones were analyzed by restriction digestion with Kpn I and Pst I, followed by agarose gel electrophoresis. A clone was isolated that encoded approximately forty-eight repeats of the elastic sequence B. Plasmid pPEP encoded the triblock copolymer protein $\mathbf{B 9}$ as a single contiguous reading frame within plasmid pET$24 a$.

Plasmid pPEP was used to transform the E. coli expression strain BL21-Gold(DE3). Large-scale fermentation (3L) was performed at $37^{\circ} \mathrm{C}$ in Terrific Broth (TB) medium supplemented with kanamycin $(50 \mu \mathrm{g} / \mathrm{mL}) .{ }^{28}$ The fermentation cultures were incubated under antibiotic selection for $36-48 \mathrm{~h}$ at $37^{\circ} \mathrm{C}$ with agitation at $175 \mathrm{rpm}$ in an orbital shaker. Cells were harvested via centrifugation at $4{ }^{\circ} \mathrm{C}$ and $4,000 \mathrm{~g}$ for $20 \mathrm{~min}$ and the cell pellet was resuspended in lysis buffer $(150 \mathrm{~mL} ; 100 \mathrm{mM} \mathrm{NaCl}, 50 \mathrm{mM}$ Tris-HCl, $\mathrm{pH} 8.0)$ and stored at $80{ }^{\circ} \mathrm{C}$. The frozen cells were lysed by three freeze/thaw cycles. Lysozyme $(1 \mathrm{mg} / \mathrm{mL})$, protease inhibitor cocktail $(5 \mathrm{~mL})$, benzonase $(25$ units $/ \mathrm{mL})$, and $\mathrm{MgCl}_{2}(1 \mathrm{mM})$ were added to the lysate and the mixture was incubated at $25^{\circ} \mathrm{C}$ for $30 \mathrm{~min}$. The cell lysate incubated for $12 \mathrm{~h}$ at $4{ }^{\circ} \mathrm{C}$ and was centrifuged at $19,000 \mathrm{~g}$ for $30 \mathrm{~min}$ at $4{ }^{\circ} \mathrm{C}$ to pellet the cell debris. The target protein was purified from the clarified cell lysate by three to five cycles of temperatureinduced precipitation $\left(4^{\circ} \mathrm{C} / 37^{\circ} \mathrm{C}\right)$ from $500 \mathrm{mM} \mathrm{NaCl}$ solution. Dialysis and lyophilization afforded protein $\mathbf{B 9}$ as a fibrous solid in an isolated yield of $418 \mathrm{mg} / \mathrm{L}$ of culture.

Amino acid compositional analysis. Calc. (mol.-\%): Ala, 8.1; Glx, 2.4; Gly, 31.9; Ile, 6.4; Pro, 20.0; Val, 31.2. Obs. (mol.-\%): Ala, 10.8; Glx, 2.0; Gly, 28.3; Ile, 7.0; Pro, 22.8; Val, 28.2. MALDI-TOF mass spectrometry, Obs. (Calc.):165,356 (165,564). Sodium 
dodecyl sulfate-polyacrylamide gel electrophoresis (SDS-PAGE) analysis indicated apparent molar masses of approximately 170,000 Da. ${ }^{29}{ }^{1} \mathrm{H}$ NMR and ${ }^{13} \mathrm{C}$ NMR spectra were measured at $4{ }^{\circ} \mathrm{C}$ and chemical shifts reported relative to the internal sodium 2,2-dimethyl-2-silapenta5-sulfonate (DSS). ${ }^{29}$

\section{Instrumentation}

Temperature-dependant solution ${ }^{1} H$ NMR spectra were recorded on INOVA 600 spectrometer (Varian, Palo Alto, CA) operating at a ${ }^{1} \mathrm{H}$ resonance frequency of $600 \mathrm{MHz}$. Thirty-two scans were acquired for signal-to noise averaging and a recycle delay of 30 seconds was used to ensure quantitative spectra. The protein was dissolved in $\mathrm{D}_{2} \mathrm{O}$ at $5{ }^{\circ} \mathrm{C}$ and NMR results were collected at defined temperatures.

Dynamic light scattering (DLS) experiments were performed using ALV Laser Goniometer, which consist in $22 \mathrm{~mW}$ HeNe linear polarized laser with $632.8 \mathrm{~nm}$ wavelength and an ALV-5000/EPP Multiple Tau Digital Correlator with 125 ns initial sampling time. Millipore water was thoroughly filtered through $0.1 \mu \mathrm{m}$ filters and directly employed for the preparation of the solutions. The samples were stabilized at temperatures ranging from $5^{\circ} \mathrm{C}$ to $40^{\circ} \mathrm{C}$ during 10 minutes prior to measurements. The accessible scattering angle range is from $30^{\circ}$ up to $140^{\circ}$. The solutions were introduced into $10 \mathrm{~mm}$ diameter glass cells. The minimum sample volume required for the experiment was 1 milliliter. The data acquisition was achieved using ALV-Correlator Control Software, and the counting time varied for each sample from $300 \mathrm{~s}$ up to $600 \mathrm{~s}$. The $\mathrm{R}_{\mathrm{H}}$ values of the micelles were obtained by a cumulant and CONTIN analysis.

Fluorescence spectra of pyrene in a protein polymer solution were acquired using an ISS PC1 (ISS, Champagne, IL) steady-state fluorescence spectrophotometer. A known amount of pyrene in acetone was added to a dry volumetric flask. After evaporating acetone, 
an aqueous solution of protein polymer was added to produce a final pyrene concentration of $6 \times 10^{-7} \mathrm{M}$.

CD spectra were obtained on a Jasco J-715 spectropolarimeter (Jasco Inc., Easton, $\mathrm{MD)}$ at a scan rate of $100 \mathrm{~nm} / \mathrm{min}$. The cell path length was $1.0 \mathrm{~mm}$ and both heating and cooling cycles were performed at $5{ }^{\circ} \mathrm{C} / \mathrm{min}$. All CD curves were smoothed using a Fourier transform noise reduction algorithm.

Attenuated total reflectance infrared spectra were acquired using a Digilab FTS 7000 (Randolph, MA) spectrometer, equipped with a narrow band $\mathrm{LN}_{2}$-cooled $\mathrm{HgCdTe}$ detector (Infrared Associates, Inc.). The spectra were recorded at various temperatures (from $-10^{\circ} \mathrm{C}$ to $65^{\circ} \mathrm{C}$ ) with 400 co-added scans at $4 \mathrm{~cm}^{-1}$ resolution using a triangular apodization function. A 50x10x2 mm germanium (Ge) crystal was mounted onto a horizontal ATR accessory (CIC Photonics, Inc.) and temperature control was achieved using a custom designed stage consisting of two thermoelectric heating/cooling (TEC) elements (Melcor Electronics), a water jacket heat sink and an AD590 temperature sensor. ${ }^{30}$ The temperature sensor and the TECs were wired to an Alpha Omega Instruments series $2 \mathrm{TC}^{2} \mathrm{TEC}$ temperature controller (Cumberland, RI) that allowed its monitoring and control to within $\pm 0.1^{\circ} \mathrm{C}$.

Background spectra of the Ge crystal were collected at every monitored temperature before sample deposition. The $10 \%$ aqueous $\mathrm{B} 9$ solution was stored frozen at $-20^{\circ} \mathrm{C}$; this solution was then thawed to $0^{\circ} \mathrm{C}$ when ready for use. A $100 \mu \mathrm{L}$ volume of the $10 \%$ aqueous B9 solution at $0^{\circ} \mathrm{C}$ was spread on the Ge crystal held at $-10^{\circ} \mathrm{C}$ and equilibrated at that temperature for approximately $10 \mathrm{~min}$ before beginning data collection. ATR spectra were collected at discrete temperatures between $-10^{\circ} \mathrm{C}$ to $65^{\circ} \mathrm{C}$. Between data points, the temperature was raised and allowed to stabilize for approximately $10 \mathrm{~min}$ before the next spectrum was collected. Spectral corrections, such as subtraction of water and baseline 
corrections, were performed using GRAMS/32 AI software (Galactic Industries Inc., Salem, $\mathrm{NH})$.

\section{2-Dimensional Correlation Analysis of Infrared Spectra}

$\beta v$ Correlation Analysis. A $\beta v$ correlation analysis is a mathematical asynchronous cross correlation performed on a set of dynamically varying IR spectra against a set of sinusoidal functions that differ only by their phase angle $\beta$, as previously described. ${ }^{31}$ The correlation analysis is mathematically described using equation (1)

$$
\Psi(v, \beta)=\frac{1}{N-1} \sum_{j=0}^{N-1} f\left(v, n_{j}\right) \sum_{k=0}^{N-1} M_{j k} \sin (k \phi+\beta)
$$

The $\beta v$ asynchronous correlation intensity $\Psi$ at some point $(v, \beta)$ represents the correlation of the measured IR spectral intensity $f\left(v, n_{j}\right)$ with the mathematical function $\sin (k \phi+\beta)$. In Equation (1), $f$ is the IR intensity at wavenumber $v, n_{j}$ is the number of the spectrum in the ordered sequence where the first spectrum number is zero, $\beta$ is the phase angle of the respective sine function, $N$ represents the total number of spectra used in the calculation, $\phi$ is a constant value in degrees (or radians) chosen based upon the total number of dynamic spectra used in the calculation, and $M_{j k}$ is the Hilbert transform matrix, which is defined in Equation (2).

$$
M_{j k}=\left\{\begin{array}{cc}
0 & i \mathrm{f} j=k \\
\frac{1}{\pi(k-j)} & \text { otherwise }
\end{array}\right.
$$

In a $\beta \nu$ correlation analysis, a new parameter, the effective phase angle $\beta_{\mathrm{e}}$, is defined as the maximum positive correlation intensity in the asynchronous $(\beta, v)$ plot for a $360^{\circ}>\beta>0^{\circ}$ range. The $\beta_{\mathrm{e}}$ value is a quantitative representation of the relative rates of change and the degree of coherence between the signal variations in a dynamic set of spectra. ${ }^{31}$ The $\beta v$ algorithms for calculating effective phase angles, as well as the plots of phase angle versus 
wavenumber were programmed using the MATLAB programming environment (Version 6, The MathWorks, Inc., Natick, MA). 


\section{DLS MEASUREMENTS: PROCEDURE TO DETERMINE THE HYDRODYNAMIC RADII.}

The DLS autocorrelation functions have been measured for protein micelles in water solutions at different concentrations from 0.05 to $0.1 \%(\mathrm{w} / \mathrm{V})$ in a temperature range $5^{\circ} \mathrm{C}<\mathrm{T}$ $<40^{\circ} \mathrm{C}$ and for angles between $30^{\circ}$ and $140^{\circ}$. The DLS correlation function was analyzed with the cumulant and CONTIN method.

When the inverse of the relaxation time $(\Gamma=1 / \tau)$ is represented as a function of the scattering vector (q), the slope corresponds to the diffusion coefficient (D) for that temperature at that concentration. All the solutions are showing monomodal distribution with a translational diffusive mode. The hydrodynamic radius $\left(\mathrm{R}_{\mathrm{H}}\right)$ can be calculated from the Stokes-Einstein equation. The variation of the hydrodynamic radius $\left(\mathrm{R}_{\mathrm{H}}\right)$ as a function of the concentration shows that the size of the micelles remains almost constant for the whole investigated range of concentration. As an example, a typical detailed analysis of the DLS experiments is presented Figure S1 for a $0.05 \%$ and Figure S2 for a $0.1 \%$ protein micelle solution. 


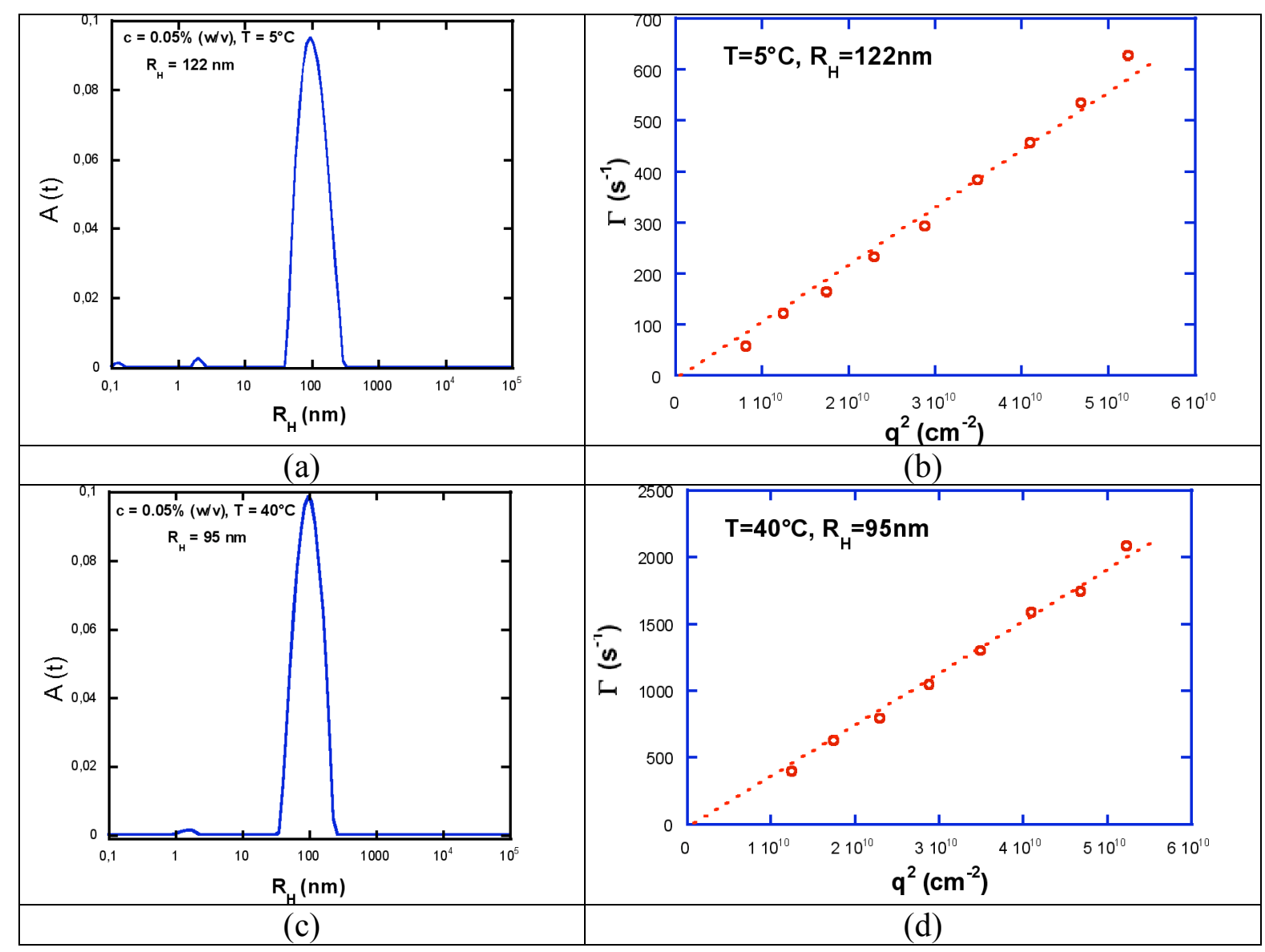

Figure S1: (a) and (c) CONTIN analysis of the correlation functions measured by DLS at $90^{\circ}$ for a protein concentration of $0.05 \%$. (b) and (d) Frequency versus $\mathrm{q}^{2}$ measured at different angles. Measurements have been performed at $5^{\circ} \mathrm{C}$ for (a) and (b), and at $40^{\circ} \mathrm{C}$ for (c) and (d). 


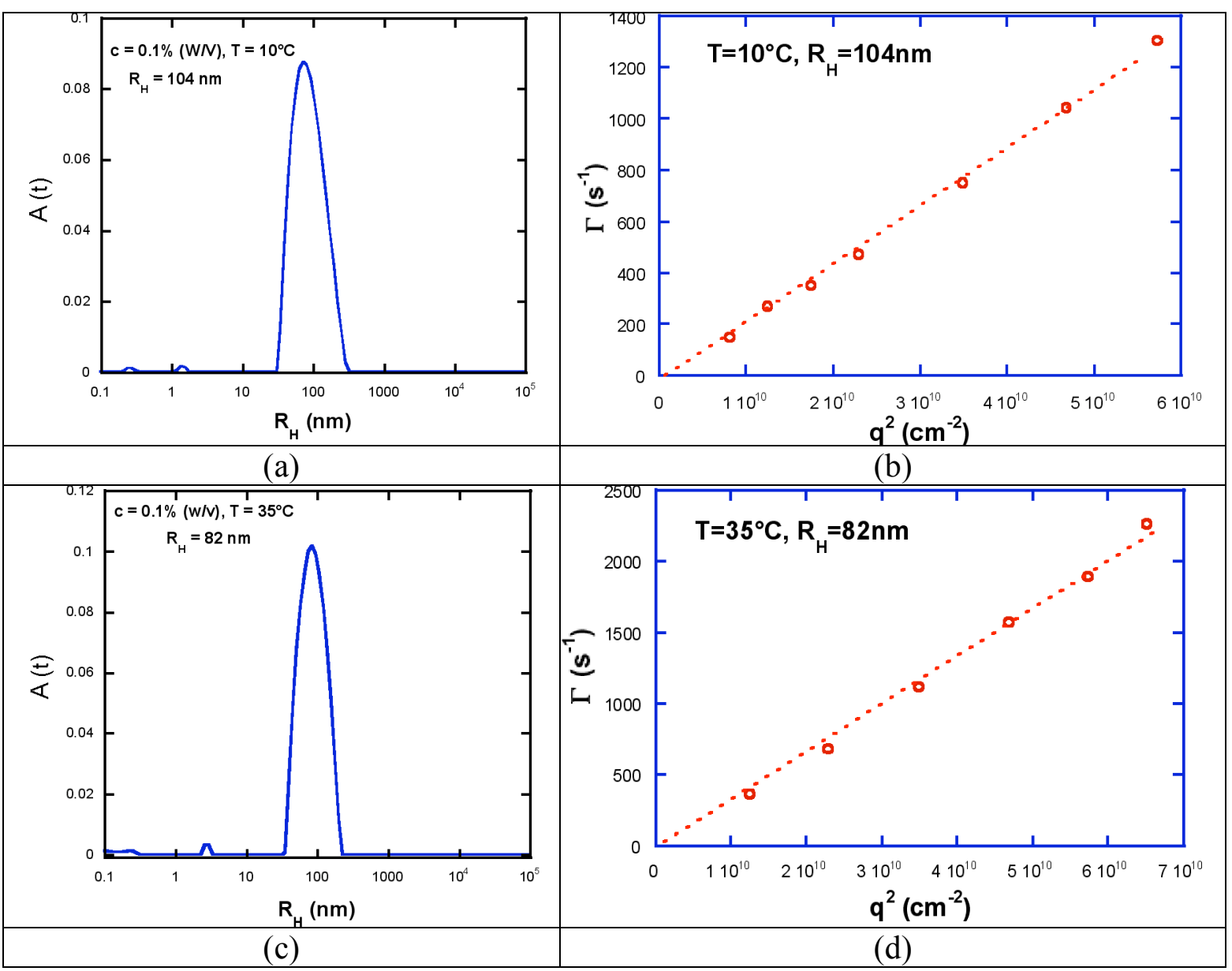

Figure S2: (a) and (c) CONTIN analysis of the correlation functions measured by DLS at $90^{\circ}$ for a protein concentration of $0.1 \%$. (b) and (d) Frequency versus $\mathrm{q}^{2}$ measured at different angles. Measurements have been performed at $10^{\circ} \mathrm{C}$ for (a) and (b), and at $35^{\circ} \mathrm{C}$ for (c) and (d). 